\title{
String corrections to circular Wilson loop and anomalies
}

\author{
Alessandra Cagnazzo, ${ }^{a, b}$ Daniel Medina-Rincon ${ }^{a, c}$ and Konstantin Zarembo ${ }^{a, b, 1}$ \\ ${ }^{a}$ Nordita, Stockholm University and KTH Royal Institute of Technology, \\ Roslagstullsbacken 23, SE-106 91 Stockholm, Sweden \\ ${ }^{b}$ Department of Physics, University of Oslo, \\ P.O. Box 1048 Blindern, N-0316 Oslo, Norway \\ ${ }^{c}$ Department of Physics and Astronomy, Uppsala University, \\ SE-751 08 Uppsala, Sweden \\ E-mail: cagnazzo@kth.se, d.r.medinarincon@nordita.org, \\ zarembo@nordita.org
}

ABSTRACT: We study string quantum corrections to the ratio of latitude and circular Wilson loops in $\mathcal{N}=4$ super-Yang-Mills theory at strong coupling. Conformal gauge for the corresponding minimal surface in $\mathrm{AdS}_{5} \times S^{5}$ is singular and we show that an IR anomaly associated with the divergence in the conformal factor removes previously reported discrepancy with the exact field-theory result. We also carefully check conformal anomaly cancellation and recalculate fluctuation determinants by directly evaluting phaseshifts for all the fluctuation modes.

Keywords: AdS-CFT Correspondence, Wilson, 't Hooft and Polyakov loops

ARXIV EPRINT: 1712.07730

\footnotetext{
${ }^{1}$ Also at ITEP, Moscow, Russia.
} 


\section{Contents}

1 Introduction 1

2 Circular Wilson loop and latitude $\quad 2$

2.1 Latitude Wilson loops 2

2.2 Classical solution 3

2.3 One-loop string corrections 4

2.4 Regularization and anomalies $\quad 5$

$\begin{array}{lll}3 & \text { Conformal anomaly cancellation } & 7\end{array}$

4 Determinants and phaseshifts $\quad 8$

4.1 Preliminaries 9

$\begin{array}{lll}4.2 & \text { Summation over frequencies } & 10\end{array}$

4.3 Phaseshifts and Jost functions 11

$\begin{array}{ll}4.4 \text { The phaseshift computation } & 12\end{array}$

$\begin{array}{lll}\text { 4.4.1 Operator } \widetilde{\mathcal{K}}_{1} & 12\end{array}$

$\begin{array}{lll}4.4 .2 & \text { Operator } \widetilde{\mathcal{K}}_{2} & 12\end{array}$

$\begin{array}{lll}4.4 .3 & \text { Operator } \widetilde{\mathcal{K}}_{3 \pm} & 12\end{array}$

$\begin{array}{ll}\text { 4.4.4 Operator } \widetilde{\mathcal{D}}_{ \pm} & 13\end{array}$

$\begin{array}{lll}4.5 & \text { Collecting the pieces together } & 15\end{array}$

$\begin{array}{lll}5 & \text { Conclusions } & 16\end{array}$

$\begin{array}{ll}\text { A Conformal anomalies } & 17\end{array}$

\section{Introduction}

We will study the ratio of two Wilson loops in $\mathcal{N}=4$ super-Yang-Mills (SYM) theory that share a common contour in space-time, but differ in their coupling to scalars, following the proposal of [1-3]. Wilson loops are important observables in gauge theories and are unique probes of the AdS/CFT correspondence since they couple directly to the string worldsheet in the dual gravitational background [4-6]. Some Wilson loops in the SYM theory can be actually computed exactly, at any coupling strength, without making any approximations. Subsequent extrapolation to strong coupling establishes a direct link between conventional QFT calculations and holography.

The simplest example of this type is the circular Wilson loop whose exact expectation value can be obtained by resumming diagrams of perturbation theory $[7,8]$ or, at a more rigorous level, by localization of the path integral on $S^{4}$ [9]. The strong-coupling extrapolation of the cirlcle agrees precisely with the area law in $\operatorname{AdS}_{5} \times S^{5}$, the result that can be 
generalized in many ways (see [10] for a review). Quite surprisingly, even the next order in the strong-coupling expansion has not been reproduced from string theory thus far, despite much effort [11-14], indicating that we do not understand in detail how strings in $\operatorname{AdS}_{5} \times S^{5}$ and other holographic backgrounds should be quantized in the Wilson-loop sector.

The difficulty lies in the defition of the measure in the string path integral and in the delicate issues with reparameterization invariance on the string worldsheet. Taking the ratio of similar Wilson loops $[1,2]$ avoids these complications, because the measure factors simply cancel. For the ratio of the latitude and the circle, considered in $[1,2]$, quantum string corrections can be computed exacty. Surprisingly, the result of the string calculation disagrees with the field-theory prediction [15]. A different quantization prescription for the string fluctuations around the latitude [3] brings the result to the agreement with field theory, but the method of [3] only applies to infinitesimally small deviations from the circle. The quantization prescritions in $[1,2]$ and in [3] differ essentially in the choice of the conformal frame on the string worldsheet, which a priori should not matter as long as the conformal anomaly cancels.

We reconsider string quantum corrections to the latitude Wilson loops, working in the same conformal frame as $[1,2]$. We pay special attention to regularization issues and ensuing anomalies and will also carefully check that the conformal anomaly cancels, which is an important consistency condition in string theory.

\section{Circular Wilson loop and latitude}

\subsection{Latitude Wilson loops}

The Wilson loop expectation value in the $\mathcal{N}=4 \mathrm{SYM}$ is defined as [4]

$$
W(C ; \mathbf{n})=\left\langle\operatorname{tr} \mathrm{P} \exp \left[i \int_{C} d \tau\left(\dot{x}^{\mu} A_{\mu}+i|\dot{x}| n^{I} \Phi_{I}\right)\right]\right\rangle,
$$

where $\Phi_{I}$ are the six scalar fields from the $\mathcal{N}=4$ supermultiplet and $\mathbf{n}$ is a unit sixdimensional vector that may change along the contour $C$. In string theory, the Wilson loop expectation value maps to the disc partition function with the boundary conditions determined by the contour $C=\left\{x^{\mu}(\tau) \mid \tau \in(0,2 \pi)\right\}$ for the embedding coordinates in $\mathrm{AdS}_{5}$, and by $\mathbf{n}(\tau)$ for $S^{5}$.

We concentrate of a particular family of Wilson loops, for which $C$ is a unit circle and $\mathbf{n}$ is a latitude of $S^{5}[15]: \mathbf{n}=\left(\sin \theta_{0} \cos \tau, \sin \theta_{0} \sin \tau, \cos \theta_{0}, \mathbf{0}\right)$. The expectation value of the latitude is known exactly $[15]:^{1}$

$$
W\left(\theta_{0}\right)=\frac{2}{\sqrt{\lambda} \cos \theta_{0}} I_{1}\left(\sqrt{\lambda} \cos \theta_{0}\right),
$$

and interpolates between the simple circle at $\theta_{0}=0$ and a supersymmetric Wilson loop with trivial expectation value [18] at $\theta_{0}=\pi / 2$. Here $\lambda=g^{2} N$ is the 't Hooft coupling of

\footnotetext{
${ }^{1}$ The latitude belongs to a more general class of supersymmetric Wilson loops which live on $S^{2} \in S^{5}$ and reduce to the effective 2d Yang-Mills theory [16] upon localization of the path integral [17]. The result quoted in the text is large- $N$ exact.
} 
the $\mathcal{N}=4$ SYM. At strong coupling,

$$
W\left(\theta_{0}\right)=\sqrt{\frac{2}{\pi \cos ^{3} \theta_{0}}} \lambda^{-\frac{3}{4}} \mathrm{e}^{\sqrt{\lambda} \cos \theta_{0}}\left(1+\mathcal{O}\left(\lambda^{-\frac{1}{2}}\right)\right) .
$$

Notice that the strong coupling and BPS $\left(\theta_{0} \rightarrow \pi / 2\right)$ limits do not commute with one another.

In string theory, the exponent in the Wilson loop vev is determined by the area of the minimal surface with the given boundary conditions, while the prefactor is a contribution of the string fluctuations and of the measure in the string path integral. Following [1-3], we consider the ratio of the circle to the latitude in which the complicated measure factor is expected to cancel. ${ }^{2}$ The field-theory prediction for the Wilson loop ratio is

$$
\Gamma \equiv \ln \frac{W(0)}{W\left(\theta_{0}\right)}=\sqrt{\lambda}\left(1-\cos \theta_{0}\right)+\frac{3}{2} \ln \cos \theta_{0}+\mathcal{O}\left(\lambda^{-1 / 2}\right) .
$$

Our goal will be to reproduce this result from the explicit one-loop calculation in string theory.

\subsection{Classical solution}

In the standard Poincaré coordinates $\left\{x^{\mu}, z\right\}$ of $\mathrm{AdS}_{5}$ and in the angular coordinates $\theta, \varphi$ of $S^{2} \subset S^{5}$, the minimal surface for the latitutde is [22]

$$
\begin{aligned}
x^{1} & =\frac{\cos \tau}{\cosh \sigma}, & x^{2} & =\frac{\sin \tau}{\cosh \sigma}, \quad z=\tanh \sigma, \\
\cos \theta & =\tanh \left(\sigma+\sigma_{0}\right), \quad \varphi & =\tau, &
\end{aligned}
$$

where $\sigma$ changes from 0 to $\infty$ and $\theta_{0}$ is related to $\sigma_{0}$ as

$$
\tanh \sigma_{0}=\cos \theta_{0}
$$

The induced worldsheet metric is given by

$$
d s^{2}=\Omega^{2}\left(d \tau^{2}+d \sigma^{2}\right)
$$

with the scale factor

$$
\Omega^{2}=\frac{1}{\sinh ^{2} \sigma}+\frac{1}{\cosh ^{2}\left(\sigma+\sigma_{0}\right)}
$$

Substituting the solution into the string action, and taking into account that the string tension is given by $\sqrt{\lambda} / 2 \pi$, in terms of the 't Hooft coupling, one gets the correct exponent in $(2.3)$.

The field-theory prediction for the next, $\mathcal{O}(1)$ term in the strong-coupling expansion of $(2.4)$ is

$$
\Gamma_{1-\text { loop }}=\frac{3}{2} \ln \tanh \sigma_{0}
$$

In string theory, this is expected to come from the one-loop quantum fluctuations of the string worldsheet [11, 23-25].

\footnotetext{
${ }^{2}$ Another way to get rid of the measure factors is to consider infinitely stretched Wilson loops and concenrate on extensive quantities. In that case an agreement between field theory and quantum corrections in string theory was obtained for the quark-anti-quark potential in the $\mathcal{N}=4 \mathrm{SYM}[19,20]$ and for the quark self-energy in the $\mathcal{N}=2^{*}$ theory [21].
} 


\subsection{One-loop string corrections}

The string oscillation modes around the classical solution (2.5) are described by the following fluctuation operators $[1-3]:^{3}$

$$
\begin{aligned}
\widetilde{\mathcal{K}}_{1}= & -\partial_{\tau}^{2}-\partial_{\sigma}^{2}+\frac{2}{\sinh ^{2} \sigma} \\
\widetilde{\mathcal{K}}_{2}= & -\partial_{\tau}^{2}-\partial_{\sigma}^{2}-\frac{2}{\cosh ^{2}\left(\sigma+\sigma_{0}\right)} \\
\widetilde{\mathcal{K}}_{3 \pm}= & -\partial_{\tau}^{2}-\partial_{\sigma}^{2} \pm 2 i\left(\tanh \left(2 \sigma+\sigma_{0}\right)-1\right) \partial_{\tau} \\
& +\left(\tanh \left(2 \sigma+\sigma_{0}\right)-1\right)\left(1+3 \tanh \left(2 \sigma+\sigma_{0}\right)\right) \\
\widetilde{\mathcal{D}}_{ \pm}= & i \partial_{\sigma} \tau_{1}-\left[i \partial_{\tau} \mp \frac{1}{2}\left(1-\tanh \left(2 \sigma+\sigma_{0}\right)\right)\right] \tau_{2} \\
& +\frac{1}{\Omega \sinh ^{2} \sigma} \tau_{3} \mp \frac{1}{\Omega \cosh ^{2}\left(\sigma+\sigma_{0}\right)},
\end{aligned}
$$

where $\tau_{i}$ are the standard Pauli matrices. The operator $\widetilde{\mathcal{K}}_{1}$ describes three string modes in $\mathrm{AdS}_{5}$, the operator $\widetilde{\mathcal{K}}_{2}$ describes three modes on $S^{5}, \widetilde{\mathcal{K}}_{3 \pm}$ arise as a result of mixing between the two remaining modes - one from the sphere, another from $\mathrm{AdS}_{5}$. The Dirac operators $\widetilde{\mathcal{D}}_{ \pm}$originate from the kinetic terms for the eight fermions remaining after kappa-symmetry gauge-fixing in the Green-Schwarz action.

The operators above are related to the ones that appear in the string action by a conformal transformation [1]:

$$
\mathcal{K}=\frac{1}{\Omega^{2}} \widetilde{\mathcal{K}}
$$

for bosons, and

$$
\mathcal{D}=\frac{1}{\Omega^{\frac{3}{2}}} \widetilde{\mathcal{D}} \Omega^{\frac{1}{2}},
$$

for fermions. The fluctuation modes of the string are naturally normalized with respect to the invariant measure of the induced metric (2.7):

$$
\left\langle\phi_{1} \mid \phi_{2}\right\rangle=\int d^{2} \sigma \sqrt{h} \phi_{1}^{\dagger} \phi_{2}=\int d \tau d \sigma \Omega^{2} \phi_{1}^{\dagger} \phi_{2},
$$

and the fluctuation operators are Hermitian with respect to this scalar product, while the tilded operators are Hermitian with respect to the usual flat measure:

$$
\left.\widehat{\left\langle\phi_{1}\right| \phi_{2}}\right\rangle=\int d \tau d \sigma \phi_{1}^{\dagger} \phi_{2}
$$

The one-loop partition function, that determines the Wilson loop expectation value, is given by the ratio of determinants of the physical, untilded operators [1]:

$$
Z\left(\sigma_{0}\right)=\frac{\operatorname{det}^{2} \mathcal{D}_{+} \operatorname{det}^{2} \mathcal{D}_{-}}{\operatorname{det}^{3 / 2} \mathcal{K}_{1} \operatorname{det}^{3 / 2} \mathcal{K}_{2} \operatorname{det}^{1 / 2} \mathcal{K}_{3+} \operatorname{det}^{1 / 2} \mathcal{K}_{3-}}
$$

\footnotetext{
${ }^{3}$ These can be obtained by specializing the general formalism of $[11,26]$ to the classical solution $(2.5)$.
} 
The Wilson loop is actually proportional to $Z\left(\sigma_{0}\right)$, but does not literally coincide with it. The string path integral contains some additional measure factors that are rather difficult to control. Fortunately, these factors do not depend on $\sigma_{0}$ and cancel in the ratio of the latitude to the circle. The one-loop free energy, if normalized as in (2.4), is given by the log-ratio of the partition functions:

$$
\Gamma_{1-\text { loop }}=\ln \frac{Z(\infty)}{Z\left(\sigma_{0}\right)} .
$$

This is the object we concentrate upon in the rest of the paper.

The tilded operators are technically easier to deal with, and in much of the previous work the conformal factors have been simply dropped. Independence on the conformal frame is a basic principle of string theory. It is thus natural to assume that the untilded operators can be seamlessly replaced by the tilded ones. However, the log-ratio of determinants computed under this assumption (which we denote by $\widetilde{\Gamma}_{1-\text { loop }}$ ) differs from the field-theory prediction (2.8) by an additional "remainder" term [1, 2]:

$$
\widetilde{\Gamma}_{1-\text { loop }}=\frac{3}{2} \ln \tanh \sigma_{0}-\frac{1}{2} \ln \frac{1+\tanh \sigma_{0}}{2} .
$$

An obvious possible cause for the discrepancy, the one that first comes to mind, is the conformal anomaly. However it was argued in [2] that the conformal anomaly is unlikely to account for the discrepancy. We refer to $[2,11]$ for technical details, and just remark that anomaly cancellation is very important in string theory. A non-zero contribution from the conformal anomaly would rather signal an internal inconsistency of the string calculation.

A caveat here is that the conformal transformation from the metric of the disc (2.7) to the flat metric of the semi-infinite cylinder changes the topology of the worldsheet and is actually singular at $\sigma=\infty$. The point $\sigma=\infty$ is a regular in the induced metric (2.7) but not in the flat metric, as illustrated in figure 1. The spectral problem for a fluctuation operator on a cylinder differs from that on a disk in an essential way and requires an IR regularization. Even if the cutoff dependence eventually cancels out, regularization may leave a finite residue. We first give a simple but not very rigorous derivation of such an IR anomaly based on elementary thermodynamics, and then proceed with a more systematic analysis of the fluctuation determinants.

\subsection{Regularization and anomalies}

The change of the conformal frame of the form (2.13) corresponds to the following chain of transformations on the determinant of $\mathcal{K}$ :

$$
\operatorname{det} \mathcal{K}=\left(\frac{\operatorname{det} \mathcal{K}}{\operatorname{det} \widetilde{\mathcal{K}}}\right)_{\text {anom. }}\left(\frac{\operatorname{det} \widetilde{\mathcal{K}}}{\operatorname{det} \widetilde{\mathcal{K}}_{\infty}}\right)_{\text {cyl. }} \operatorname{det} \widetilde{\mathcal{K}}_{\infty}
$$

where $\widetilde{\mathcal{K}}_{\infty}$ is the asymptotic operator obtained by taking $\sigma \rightarrow \infty$ in $(2.9)-(2.11)$, which is just the free Klein-Gordon operator:

$$
\widetilde{\mathcal{K}}_{\infty}=-\partial_{\tau}^{2}-\partial_{\sigma}^{2},
$$




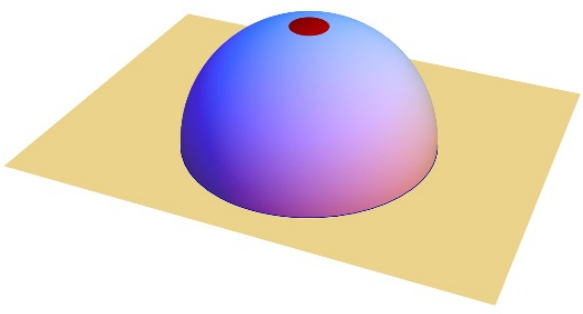

(a)

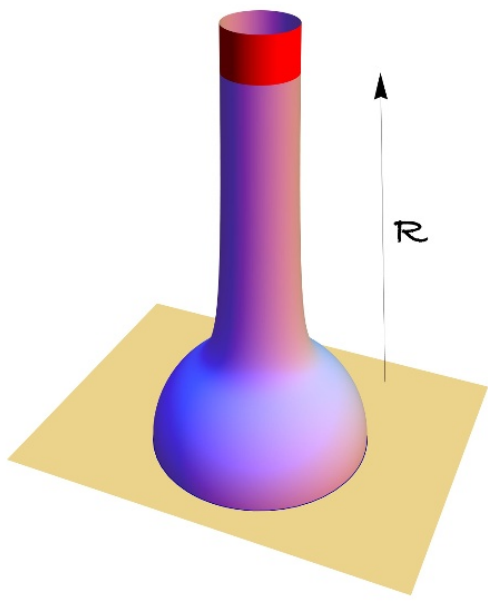

(b)

Figure 1. Schematic representation of the induced metric on the minimal surface (a), and of the flat coordinates on the cylinder (b). The conformal transformation between the two is singular at the symmetry point of the minimal surface $(\sigma=\infty)$. The region $\sigma>R$, removed by regularization, maps to a small circle of area $s$ on the minimal surface in the target space.

or the free Dirac operator, in case of fermions:

$$
\widetilde{\mathcal{D}}_{\infty}=i \tau_{1} \partial_{\sigma}-i \tau_{2} \partial_{\tau}
$$

The first ratio in (2.20) is the conformal anomaly, the second one is well-defined on a cylinder, while separately $\operatorname{det} \widetilde{\mathcal{K}}$ and $\operatorname{det} \widetilde{\mathcal{K}}_{\infty}$ require an IR regularization. The IR cutoff is manifestly necessary for the Gelfand-Yaglom method used in [1,2], and is implicit in a more direct phase-shift calculation that will be carried out in section 4 .

The standard way to regularize the problem is to impose the Dirichlet boundary conditions on the wavefunction of $\widetilde{\mathcal{K}}$ (or $\widetilde{\mathcal{K}}_{\infty}$ ) at some large but finite $\sigma=R$. This corresponds to removing a small segment of the minimal surface shown as a red circle in figure 1a. This is not such an innocent procedure, as can be seem by comparing determinants of the Laplacian on a disk and on a disk with a small hole [27]. Even though the IR cutoff cancels in the final answer, intermediate steps do depend on $R$. At the same time, the cutoff $R$ does not have any invariant meaning by itself. To faithfully compare partition functions at different values of $\sigma_{0}$, we need a diffeomorphism-invariant regularization.

As an invariant regularization parameter we can take the area of the segment removed from the minimal surface when Dirichlet boundary conditions are imposed at $\sigma=R$ :

$$
s=\int_{\sigma>R} d^{2} \sigma \sqrt{h}=2 \pi \int_{R}^{\infty} d \sigma \Omega^{2} \simeq 4 \pi\left(1+\mathrm{e}^{-2 \sigma_{0}}\right) \mathrm{e}^{-2 R} .
$$

The coordinate-dependent cutoff is related to the invariant one as

$$
R=\frac{1}{2} \ln \frac{8 \pi}{s\left(1+\tanh \sigma_{0}\right)} \equiv R_{\mathrm{inv}}-\frac{1}{2} \ln \frac{1+\tanh \sigma_{0}}{2} .
$$


Because $R$ is a coordinate-dependent quantity with no invariant meaning, in comparing the partition functions at different $\sigma_{0}$, it is $R_{\text {inv }}$ rather than $R$ that should be kept fixed. Diffeomorphism-invariant regularization implies that $R$ has to dependent on $\sigma_{0}$.

The partition function depends on $R$ through the last factor in $(2.20)$ and since $\widetilde{\mathcal{K}}_{\infty}$ and $\widetilde{\mathcal{D}}_{\infty}$ are just the free Klein-Gordon and Dirac opertors, the asymptotic contribution to the partition function is given by the free energy of a gas of free particles in $1+1$ dimensions, given by

$$
F=-\left(2 N_{b}+N_{f}\right) \frac{\pi}{12} T^{2} V
$$

In our case $N_{b}=8=N_{f}, T=1 /(2 \pi)$ and $V=R$. We thus have

$$
\ln \widetilde{Z}_{\infty}=-\frac{F}{T}=R
$$

The IR divergence cancels in the ratio (2.18), but leaves a finite, $\sigma_{0}$-dependent remnant due to $(2.24)$ :

$$
\widetilde{\Gamma}_{\infty}=-R\left(\sigma_{0}\right)+R(\infty)=\frac{1}{2} \ln \frac{1+\tanh \sigma_{0}}{2} .
$$

Combined with (2.19), this gives

$$
\widetilde{\Gamma}_{1-\text { loop }}+\widetilde{\Gamma}_{\infty}=\frac{3}{2} \ln \tanh \sigma_{0},
$$

which agrees with the localization prediction (2.8).

This is the main result of the paper. To validate this result we need to check that the conformal anomaly cancels, which we do in the next section. Later we will also reanalyze the partition function on the cylinder and will derive the above result by a direct spectral analysis of the fluctuation operators.

\section{Conformal anomaly cancellation}

The anomaly contribution to the free energy is

$$
\Gamma_{\text {anom }}=\frac{1}{2} \sum_{a}(-1)^{F_{a}} \ln \frac{\operatorname{det} \mathcal{K}_{a}}{\operatorname{det} \widetilde{\mathcal{K}}_{a}}
$$

where the summation runs over all operators in (2.17) with the appropriate multiplicities. The operators in the numerator and denominator differ by the conformal factor, and if one were allowed to factorize the determinants, the sum would trivially vanish. The story is more complicated because of the need in the intermediate UV regularization.

The anomaly, being a local effect of UV divergences, can be computed by the standard DeWitt-Seeley expansion. For completeness we give a brief derivation of the conformal anomaly adpated to our case in the appendix A. The results (A.10) and (A.15) directly apply to the operators (2.9)-(2.14), upon bringing them to the standard Klein-Gordon/Dirac 
form (A.1), (A.2), with the identifications

$$
\begin{aligned}
\phi & =-\ln \Omega \\
E_{1} & =\frac{2}{\sinh ^{2} \sigma} \\
E_{2} & =-\frac{2}{\cosh ^{2}\left(\sigma+\sigma_{0}\right)} \\
E_{3 \pm} & =-\frac{2}{\cosh ^{2}\left(2 \sigma+\sigma_{0}\right)} \\
a_{ \pm} & =\frac{1}{\Omega \sinh ^{2} \sigma} \\
v_{ \pm} & =\mp \frac{1}{\Omega \cosh ^{2}\left(\sigma+\sigma_{0}\right)} .
\end{aligned}
$$

First we notice that the boundary terms in the anomaly trivially cancel between bosons and fermions, just by matching the number of degrees of freedom. To see that the bulk anomaly also cancels it is convenient to bring the scale factor of the metric to the following form:

$$
\Omega^{2}=\frac{\cosh \sigma_{0} \cosh \left(2 \sigma+\sigma_{0}\right)}{\sinh ^{2} \sigma \cosh ^{2}\left(\sigma+\sigma_{0}\right)}
$$

from which it immediately follows that

$$
\partial_{\sigma}^{2} \phi=-\partial_{\sigma}^{2} \ln \Omega=\frac{1}{\cosh ^{2}\left(\sigma+\sigma_{0}\right)}-\frac{1}{\sinh ^{2} \sigma}-\frac{2}{\cosh ^{2}\left(2 \sigma+\sigma_{0}\right)} .
$$

This enters the anomaly with a prefactor

$$
\left(\frac{1}{6} \times 8+\frac{1}{12} \times 8\right) \phi=2 \phi
$$

On the other hand,

$$
\begin{gathered}
3 E_{1}+3 E_{2}+E_{3+}+E_{3-}+4\left(v_{+}^{2}-a_{+}^{2}\right)+4\left(v_{-}^{2}-a_{-}^{2}\right) \\
=\frac{2}{\cosh ^{2}\left(\sigma+\sigma_{0}\right)}-\frac{2}{\sinh ^{2} \sigma}-\frac{4}{\cosh ^{2}\left(2 \sigma+\sigma_{0}\right)},
\end{gathered}
$$

and the two terms in the anomaly completely compensate one another.

The anomaly thus cancels in the partition function (2.17) at each value of $\sigma_{0}$, not only in the ratio, as actually expected.

\section{Determinants and phaseshifts}

The fluctuation determinants were evaluated in [1,2] with the help of the Gelfand-Yaglom method. Here we recalculate them by a more direct approach, evaluating phaseshifts for each operator and then integrating over the phase space of string fluctuations. First we set up the general scheme for the phaseshift computation and then apply it to each operator in turn. 


\subsection{Preliminaries}

The operators at hand have the general form (we start with bosons, for fermions the same scheme works with minor modifications):

$$
\widetilde{\mathcal{K}}=-\partial_{\sigma}^{2}+V\left(\partial_{\tau}, \sigma\right) .
$$

The asymptotics at infinity are that of the free d'Alambert operator:

$$
V\left(\partial_{\tau}, \infty\right)=-\partial_{\tau}^{2}
$$

The Fourier expansion in $\tau$ replaces $\partial_{\tau}$ by $-i \omega$, with integer frequency, or half-integer depending on whether the boundary conditions are periodic or anti-periodic in the $\tau$ direction. The spectrum thus decomposes into a sequence of one-dimensional problems for each Fourier mode:

$$
\left(-\partial_{\sigma}^{2}+V(-i \omega, \sigma)\right) \Psi=\Lambda \Psi .
$$

The boundary condition at $\sigma=0$ is

$$
\Psi(0)=0 .
$$

After the boundary condition is imposed the wavefunction is fixed up to normalization. Since the potential vanishes at infinity, the wavefunction asymptotically has an oscillating behavior:

$$
\Psi(\sigma) \stackrel{\sigma \rightarrow \infty}{\simeq} \mathrm{C} \sin (p \sigma+\delta) .
$$

The eigenvalue can be read off the asymptotic form of the Schrödinger equation (4.3):

$$
\Lambda=\omega^{2}+p^{2} .
$$

To define the determinant of an operator with a continuous spectrum we need to introduce an IR cutoff by imposing another boundary condition at $\sigma=R$ :

$$
\Psi(R)=0 .
$$

The spectrum then becomes discrete due to momentum quantization condition:

$$
p_{n} R+\delta\left(\omega, p_{n}\right) \simeq \pi n,
$$

which follows from the asymptotic form of the wavefunction (4.5) and is thus valid as long as $R$ is much larger than the range of the potential in the Schrödinger equation. The density of states $\rho=\partial n / \partial p$ in the limit of $R \rightarrow \infty$ hence takes the form

$$
\rho(p)=\frac{1}{\pi}\left(R+\frac{\partial \delta(\omega, p)}{\partial p}\right) .
$$

Often omitted extensive piece proportional to $R$ has to be kept here, since it only cancels in the ratio of the two partition functions at different $\sigma_{0}$ and the cutoff $R$ as we have seen depends on $\sigma_{0}$ if regularization is to preserve general covariance. 


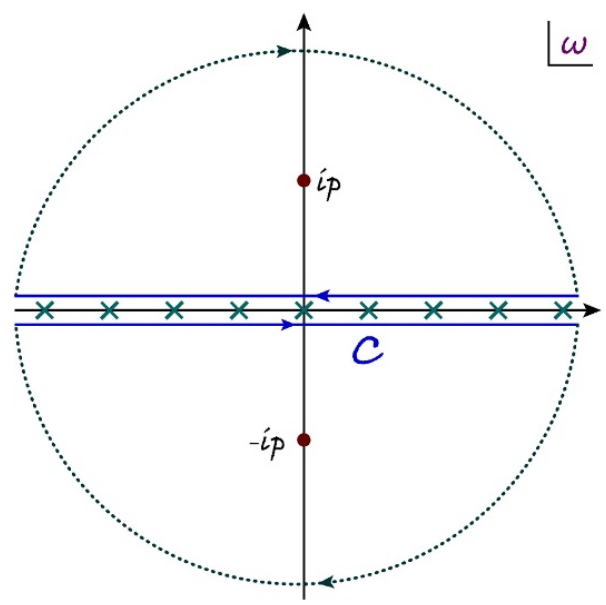

Figure 2. Contours of integration in the complex frequency plane.

The determinant of $\widetilde{\mathcal{K}}$ is obtained by multiplying all the eigenvalues:

$$
\begin{aligned}
\ln \operatorname{det} \widetilde{\mathcal{K}} & =\sum_{\omega} \int_{0}^{\infty} \frac{d p}{\pi}\left(R+\frac{\partial \delta(\omega, p)}{\partial p}\right) \ln \left(\omega^{2}+p^{2}\right) \\
& =-\sum_{\omega} \int_{0}^{\infty} \frac{d p}{\pi} \frac{2 p}{\omega^{2}+p^{2}}(\delta(\omega, p)+R p),
\end{aligned}
$$

with $\omega \in \mathbb{Z}$ or $\omega \in \mathbb{Z}+1 / 2$ for periodic/anti-periodic boundary conditions.

Our strategy will be to directly evaluate phaseshifts for all the operators, sum over frequencies and integrate over spacial momenta. Before proceeding with explicit calculations we sum over the Matsubara frequency using standard tools of Statistical Mechanics [28], and make a few technical remarks that streamline calculation of the phaseshifts.

\subsection{Summation over frequencies}

The standard trick is to replace summation by integration along the contour shown in figure 2:

$$
\ln \operatorname{det} \widetilde{\mathcal{K}}=-\int_{C} \frac{d \omega}{2 \pi i} \cot \pi \omega \int_{0}^{\infty} d p \frac{2 p}{\omega^{2}+p^{2}}(\delta(\omega, p)+R p) .
$$

The poles of the cotangent recover the sum over the Matsubara frequencies.

Assuming that the phaseshift does not grow too fast at large frequencies (in the simplest cases the phaseshift does not depend on the frequency at all), the contour of integration can be closed in the upper and lower half-planes, as shown in figure 2 , after which the integral over $\omega$ picks up residues at $\omega= \pm i p$. Denoting

$$
\delta_{ \pm}(p) \equiv \delta( \pm i p, p)
$$

we get for the determinant:

$$
\ln \operatorname{det} \widetilde{\mathcal{K}}=-\int_{0}^{\infty} d p \operatorname{coth} \pi p\left(\delta_{+}(p)+\delta_{-}(p)+2 R p\right) .
$$


This equation expresses the determinant entirely through the on-shell data. It suffices to solve the Schrödinger equation (4.3) at $\omega= \pm i p$ and $\Lambda=0$.

If the boundary conditions are anti-periodic and the Matsubara frequencies $\omega$ are halfinteger, the summation formulas differ by substitutions cot $\rightarrow-\tan$, coth $\rightarrow \tanh$ :

$$
\ln \operatorname{det} \widetilde{\mathcal{K}}_{F}=-\int_{0}^{\infty} d p \tanh \pi p\left(\delta_{+}(p)+\delta_{-}(p)+2 R p\right) .
$$

Normally, the particle and anti-particle phaseshifts $\delta_{+}$and $\delta_{-}$are equal, but some operators that we encounter have a spectral asymmetry resulting in different density of states for particles and anti-particles.

\subsection{Phaseshifts and Jost functions}

Instead of solving the Schrödinger equation with the correct boundary conditions (4.4) it is sometimes easier to find the Jost functions, which are the solutions that asymptote to unit-normalized plane waves at infinity:

$$
Y_{p}(\sigma) \stackrel{\sigma \rightarrow \infty}{\simeq} \mathrm{e}^{i p \sigma}, \quad \bar{Y}_{p}(\sigma) \stackrel{\sigma \rightarrow \infty}{\simeq} \mathrm{e}^{-i p \sigma}
$$

For a self-adjoint Schrödinger operator with a real potential the Jost functions are complex conjugate to one another: $\bar{Y}_{p}=Y_{p}^{*}$, but if the potential is complex, which is the case for the operators $\widetilde{\mathcal{K}}_{3 \pm}$ for example, then the two Jost functions are not related in any simple way.

The Jost functions form a complete set of solutions to the Schrödinger equation. The solution that satisfy the correct boundary conditions is a linear combination of the two Jost functions:

$$
\Psi_{p}(\sigma)=\bar{Y}_{p}(0) Y_{p}(\sigma)-Y_{p}(0) \bar{Y}_{p}(\sigma) .
$$

This linear combination indeed vanishes at $\sigma=0$. Comparing its behaviour at infinity with (4.5), we find:

$$
\bar{Y}_{p}(0) \mathrm{e}^{i p \sigma}-Y_{p}(0) \mathrm{e}^{-i p \sigma}=\frac{\mathrm{C}}{2 i} \mathrm{e}^{i p \sigma+\delta}-\frac{\mathrm{C}}{2 i} \mathrm{e}^{-i p \sigma-\delta},
$$

which expressed the phaseshift through the Jost data:

$$
\frac{\bar{Y}_{p}(0)}{Y_{p}(0)}=\mathrm{e}^{2 i \delta} .
$$

In the self-adjoint case the Jost functions are complex conjugate and their ratio is a pure phase. The phaseshift is real in this case. If the Schrödinger operator is not selfadjoint, the phaseshift may have an imaginary part. In general,

$$
\delta(p)=\frac{i}{2} \ln \frac{Y_{p}(0)}{\bar{Y}_{p}(0)} .
$$

We will use this formula to evaluate the phaseshifts of the fluctuation operators for the latitude by explicitly calculating the Jost functions in each case. The same scheme can be applied to fermions with minor modifications related to their two-component nature. 


\subsection{The phaseshift computation}

\subsubsection{Operator $\widetilde{\mathcal{K}}_{1}$}

The differential equation for this operator is given by

$$
\left(-\partial_{\sigma}^{2}+\frac{2}{\sinh ^{2} \sigma}\right) \chi_{1}=p^{2} \chi_{1}
$$

The solutions to this equation are given by the Jost functions

$$
Y_{p}(\sigma)=\mathrm{e}^{i p \sigma} \frac{i p-\operatorname{coth} \sigma}{i p-1}, \quad \bar{Y}_{p}(\sigma)=\mathrm{e}^{-i p \sigma} \frac{i p+\operatorname{coth} \sigma}{i p+1},
$$

satisfying $Y_{p}=\left(\bar{Y}_{p}\right)^{*}$ and $\bar{Y}_{p}=\left(Y_{p}\right)^{*}$. Using equation (4.19), we obtain

$$
\delta_{1}=\frac{i}{2} \ln \frac{i p+1}{i p-1}=\frac{\pi}{2}-\arctan p .
$$

\subsubsection{Operator $\widetilde{\mathcal{K}}_{2}$}

The corresponding differential equation is given by

$$
\left(-\partial_{\sigma}^{2}-\frac{2}{\cosh ^{2}\left(\sigma+\sigma_{0}\right)}\right) \chi_{2}=p^{2} \chi_{2} .
$$

In this case, the Jost functions are

$$
Y_{p}=\mathrm{e}^{i p \sigma} \frac{i p-\tanh \left(\sigma+\sigma_{0}\right)}{i p-1}, \quad \bar{Y}_{p}=\mathrm{e}^{-i p \sigma} \frac{i p+\tanh \left(\sigma+\sigma_{0}\right)}{i p+1},
$$

satisfying $Y_{p}=\left(\bar{Y}_{p}\right)^{*}$ and $\bar{Y}_{p}=\left(Y_{p}\right)^{*}$. From equation (4.19), we have

$$
\delta_{2}=\frac{i}{2} \ln \left(\frac{1+i p}{1-i p} \frac{\tanh \sigma_{0}-i p}{\tanh \sigma_{0}+i p}\right)=-\arctan p+\arctan \frac{p}{\tanh \sigma_{0}} .
$$

\subsubsection{Operator $\widetilde{\mathcal{K}}_{3 \pm}$}

The Schrödinger problem for $\widetilde{\mathcal{K}}_{3+}$ is

$$
\left[-\partial_{\sigma}^{2}+\left(3 \tanh \left(2 \sigma+\sigma_{0}\right)+1 \pm 2 i p\right)\left(\tanh \left(2 \sigma+\sigma_{0}\right)-1\right)\right] \psi_{p}=p^{2} \psi_{p}
$$

where we have set $\partial_{\tau} \rightarrow-i \omega= \pm p$. The \pm sign refers to particle/anti-particle modes.

The potential in the Schrödinger equation is of the solvable Rosen-Morse type. The solution $^{4}$ can be found by the substitution [29]

$$
x=\frac{1-\tanh \left(2 \sigma+\sigma_{0}\right)}{2}
$$

\footnotetext{
${ }^{4}$ The Rosen-Morse potential is solvable in hypergepmetric functions for any values of the frequency $\omega$, not necessarily on-shell [29]. Using this general solution an analytic expression for the off-shell phaseshift $\delta(\omega, p)$ can be found for any $\omega$ and $p$. We do not display this more general function here, because we only need the on-shell phaseshifts $\delta_{ \pm}(p)$ to compute the determinant.
} 
accompanied by the following ansatz for the wavefunction:

$$
\psi_{p}(\sigma)=\mathrm{e}^{\mp i p \sigma+\sigma} \cosh ^{-\frac{1}{2}}\left(2 \sigma+\sigma_{0}\right) \chi(x)
$$

which leads to

$$
\left[\frac{d}{d x} x(1-x) \frac{d}{d x}-\left(x \mp \frac{i p}{2}\right) \frac{d}{d x}\right] \chi=0
$$

or

$$
\psi_{p}(\sigma)=\mathrm{e}^{ \pm i p \sigma-\sigma} \cosh ^{\frac{1}{2}}\left(2 \sigma+\sigma_{0}\right) \tilde{\chi}(x),
$$

which leads to

$$
\left[\frac{d}{d x} x(1-x) \frac{d}{d x}+\left(x \mp \frac{i p}{2}\right) \frac{d}{d x}+1\right] \tilde{\chi}=0 .
$$

These equations have simple solutions:

$$
\chi(x)=1, \quad \tilde{\chi}(x)=x-1 \pm \frac{i p}{2} .
$$

From here we find the Jost functions:

$$
\begin{aligned}
Y_{p}^{+}(\sigma) & =\mathrm{e}^{i p \sigma-\sigma-\frac{\sigma_{0}}{2}} \sqrt{2 \cosh \left(2 \sigma+\sigma_{0}\right)} \frac{1-i p+\tanh \left(2 \sigma+\sigma_{0}\right)}{2-i p}, \\
\bar{Y}_{p}^{+}(\sigma) & =\frac{\mathrm{e}^{-i p \sigma+\sigma+\frac{\sigma_{0}}{2}}}{\sqrt{2 \cosh \left(2 \sigma+\sigma_{0}\right)}}, \\
Y_{p}^{-} & =\left(\bar{Y}_{p}^{+}\right)^{*}, \quad \bar{Y}_{p}^{-}=\left(Y_{p}^{+}\right)^{*} .
\end{aligned}
$$

The Jost functions $Y_{p}$ and $\bar{Y}_{p}$ are not complex conjugate to one another, because the potential is complex, and consequently the phaseshifts will have an imaginary part. There is also a spectral asymmetry between particle and anti-particle phaseshifts, but it is easy to quantify it because particle and anti-particle Jost functions are related by complex conjugation: $\delta_{-}(p)^{*}=\delta_{+}(p)$. Taking into account the last equation in (4.33), we get from (4.19):

$$
\delta_{3+}(p)+\delta_{3-}(p)=\frac{i}{2} \ln \frac{Y_{p}^{+}(0) \bar{Y}_{p}^{+}(0)^{*}}{Y_{p}^{+}(0)^{*} \bar{Y}_{p}^{+}(0)}=\arctan \frac{p}{1+\tanh \sigma_{0}}-\arctan \frac{p}{2} .
$$

The answer for $\widetilde{\mathcal{K}}_{3-}$ is the same up to exchanging $\delta_{3+}$ with $\delta_{3-}$.

\subsubsection{Operator $\widetilde{\mathcal{D}}_{ \pm}$}

It is convenient to consider, instead of $\widetilde{\mathcal{D}}_{\alpha}$, the eigenvalue problem for $i \tau_{2} \widetilde{\mathcal{D}}_{\alpha}$. The index $\alpha$ that takes values \pm is introduced here in order to distinguish the operator label from the particle/anti-particle index. The spectral problem for the resulting Dirac operator takes the form:

$$
\left(i \tau_{3} \partial_{\sigma}+\frac{i \alpha}{2}\left[1-\tanh \left(2 \sigma+\sigma_{0}\right)\right] \mathbb{1}-\frac{1}{\Omega \sinh ^{2} \sigma} \tau_{1}-i \frac{\alpha}{\Omega \cosh ^{2}\left(\sigma+\sigma_{0}\right)} \tau_{2}\right) \chi_{\alpha}=\mp p \chi_{\alpha}
$$


where the $\mp$ sign comes from the two choices of closing the integration contour described in figure 2.

The most general solution of the Dirac equation is a superposition of the Jost functions

$$
\begin{aligned}
& Y_{p, \alpha}^{ \pm}=e^{ \pm \alpha i p \sigma}\left(\delta_{\alpha,+}\left(\begin{array}{c}
c_{\mathrm{I}} \\
c_{\mathrm{II}}
\end{array}\right)+\delta_{\alpha,-}\left(\begin{array}{c}
c_{\mathrm{II}} \\
c_{\mathrm{I}}
\end{array}\right)\right), \\
& \bar{Y}_{p, \alpha}^{ \pm}=e^{\mp \alpha i p \sigma}\left(\delta_{\alpha,+}\left(\begin{array}{c}
\bar{c}_{\mathrm{I}} \\
\bar{c}_{\mathrm{II}}
\end{array}\right)+\delta_{\alpha,-}\left(\begin{array}{c}
\bar{c}_{\mathrm{II}} \\
\bar{c}_{\mathrm{I}}
\end{array}\right)\right),
\end{aligned}
$$

where

$$
\begin{aligned}
c_{\mathrm{I}}= & \frac{1}{\left( \pm \alpha i p-\frac{3}{2}\right)\left( \pm \alpha i p-\frac{1}{2}\right)} \frac{2^{-7 / 4} e^{\sigma / 2} e^{-5 \sigma_{0} / 4} \Omega^{1 / 2}}{\cosh ^{1 / 4} \sigma_{0} \sqrt{\sinh \sigma \cosh \left(\sigma+\sigma_{0}\right)}} \\
& {\left[e^{-3 \sigma}\left(p^{2}+\frac{1}{4}\right)+\left( \pm \alpha i p-\frac{3}{2}\right)\left(e^{-\sigma}\left( \pm \alpha i p+\frac{1}{2}\right)+2 e^{2 \sigma_{0}} \sinh \sigma\left( \pm \alpha i p-\frac{\operatorname{coth} \sigma}{2}\right)\right)\right], } \\
c_{\mathrm{II}}= & \frac{i \alpha}{\left( \pm \alpha i p-\frac{3}{2}\right)\left( \pm \alpha i p-\frac{1}{2}\right)} \frac{2^{-7 / 4} e^{-\sigma / 2} e^{-\sigma_{0} / 4} \Omega}{\cosh \sigma_{0} \cosh ^{3 / 4}\left(2 \sigma+\sigma_{0}\right)} \\
& {\left[\left( \pm \alpha i p-\frac{1}{2}\right)\left(2+\cosh \left(2\left(\sigma+\sigma_{0}\right)\right)-\cosh (2 \sigma)\right)-\sinh \left(2\left(\sigma+\sigma_{0}\right)\right)+\sinh (2 \sigma)\right], } \\
\bar{c}_{\mathrm{I}}= & \frac{i \alpha}{\left( \pm \alpha i p+\frac{1}{2}\right)} \frac{e^{\sigma / 2} e^{\sigma_{0} / 4} \Omega}{2^{5 / 4} \cosh ^{1 / 4}\left(2 \sigma+\sigma_{0}\right)}, \\
\bar{c}_{\mathrm{II}}= & \frac{1}{\left( \pm \alpha i p+\frac{1}{2}\right)} \frac{e^{\sigma / 2} e^{\sigma_{0} / 4}}{2^{1 / 4} \cosh ^{1 / 4}\left(2 \sigma+\sigma_{0}\right)}\left( \pm \alpha i p+\frac{1}{2}\left(\frac{\cosh \left(2 \sigma+\sigma_{0}\right)}{\sinh \sigma \cosh \left(\sigma+\sigma_{0}\right)}-1\right)\right) .
\end{aligned}
$$

Asymptotically, the two solutions behave as

$$
\begin{aligned}
& \lim _{\sigma \rightarrow \infty} Y_{p, \alpha}^{ \pm}=e^{ \pm \alpha i p \sigma}\left(\delta_{\alpha,+}\left(\begin{array}{l}
1 \\
0
\end{array}\right)+\delta_{\alpha,-}\left(\begin{array}{l}
0 \\
1
\end{array}\right)\right), \\
& \lim _{\sigma \rightarrow \infty} \bar{Y}_{p, \alpha}^{ \pm}=e^{\mp \alpha i p \sigma}\left(\delta_{\alpha,+}\left(\begin{array}{l}
0 \\
1
\end{array}\right)+\delta_{\alpha,-}\left(\begin{array}{l}
1 \\
0
\end{array}\right)\right) .
\end{aligned}
$$

Close to $\sigma=0$, the Yost functions behave as

$$
\begin{aligned}
& \lim _{\sigma \rightarrow 0} Y_{p, \alpha}^{ \pm}=\frac{v_{\alpha}^{ \pm}}{\sigma}\left(\delta_{\alpha,+}\left(\begin{array}{c}
i \\
1
\end{array}\right)+\delta_{\alpha,-}\left(\begin{array}{c}
1 \\
-i
\end{array}\right)\right)+\mathcal{O}(\sigma), \\
& \lim _{\sigma \rightarrow 0} \bar{Y}_{p, \alpha}^{ \pm}=\frac{\bar{v}_{\alpha}^{ \pm}}{\sigma}\left(\delta_{\alpha,+}\left(\begin{array}{c}
i \\
1
\end{array}\right)+\delta_{\alpha,-}\left(\begin{array}{c}
1 \\
-i
\end{array}\right)\right)+\mathcal{O}(\sigma),
\end{aligned}
$$

where

$$
v_{\alpha}^{ \pm}=i \alpha \frac{\cosh ^{1 / 4} \sigma_{0}}{2^{3 / 4} e^{\sigma_{0} / 4}} \frac{ \pm \alpha i p-\frac{1}{2}-\tanh \sigma_{0}}{\left( \pm \alpha i p-\frac{3}{2}\right)\left( \pm \alpha i p-\frac{1}{2}\right)}, \quad \bar{v}_{\alpha}^{ \pm}=\frac{e^{\sigma_{0} / 4}}{2^{5 / 4} \cosh ^{1 / 4} \sigma_{0}} \frac{1}{ \pm \alpha i p+\frac{1}{2}} .
$$

From the above, it is easy to see that the superposition

$$
\chi_{\alpha}^{ \pm}=\bar{v}_{\alpha}^{ \pm} Y_{p, \alpha}^{ \pm}-v_{\alpha}^{ \pm} \bar{Y}_{p, \alpha}^{ \pm}
$$


vanishes for $\sigma \rightarrow 0$, and therefore satisfies the right boundary conditions at $\sigma=0$. At $\sigma \rightarrow \infty$, the correct solution behaves as

$$
\chi_{\alpha}^{ \pm} \simeq \delta_{\alpha,+}\left(\begin{array}{c}
\bar{v}_{+}^{ \pm} \mathrm{e}^{ \pm i p \sigma} \\
-v_{+}^{ \pm} \mathrm{e}^{\mp i p \sigma}
\end{array}\right)+\delta_{\alpha,-}\left(\begin{array}{c}
-v_{-}^{ \pm} \mathrm{e}^{ \pm i p \sigma} \\
\bar{v}_{-}^{ \pm} \mathrm{e}^{\mp i p \sigma}
\end{array}\right) .
$$

To define the fermion phaseshift we need to understand what replaces the auxiliary boundary condition (4.7) for fermions. Since the Dirac equation is of the first order it is impossible to set both spinor components of the wavefunction to zero. Only a chiral projection of the wavefunction can vanish. Choosing the chirality condition (any other choice leads to equivalent results) as

$$
\tau_{2} \chi(R)=\chi(R),
$$

we get the momentum quantization condition in the form (4.8) with

$$
\delta_{\alpha}^{ \pm}= \pm \frac{\alpha}{2} \operatorname{Arg}\left(\frac{\bar{v}_{\alpha}^{ \pm}}{v_{\alpha}^{ \pm}}\right)=\frac{\pi}{2}+\frac{1}{2} \arctan \frac{p}{\frac{1}{2}+\tanh \sigma_{0}}-\arctan 2 p-\frac{1}{2} \arctan \frac{2 p}{3} .
$$

\subsection{Collecting the pieces together}

Expressing the determinants in (2.17) through the on-shell phaseshifts with the help of (4.13) and (4.14), and collecting all the pieces together we get for the log of the partition function:

$$
\begin{aligned}
\ln Z\left(\sigma_{0}\right)= & \int_{0}^{\infty} d p\left[\operatorname { c o t h } \pi p \left(\arctan \frac{p}{1+\tanh \sigma_{0}}+3 \arctan \frac{p}{\tanh \sigma_{0}}-\arctan \frac{p}{2}\right.\right. \\
& \left.-6 \arctan p+\frac{3 \pi}{2}\right)-4 \tanh \pi p\left(\arctan \frac{p}{\frac{1}{2}+\tanh \sigma_{0}}-2 \arctan 2 p\right. \\
& \left.\left.+\arctan \frac{2 p}{3}+\pi\right)+8 R p(\operatorname{coth} \pi p-\tanh \pi p)\right]
\end{aligned}
$$

The easiest way to compute the integral is by differentiation in $\sigma_{0}$ :

$$
\begin{aligned}
\frac{d}{d \sigma_{0}} \ln Z\left(\sigma_{0}\right)= & \frac{1}{\cosh ^{2} \sigma_{0}} \int_{0}^{\infty} d p p\left[\frac{4 \tanh \pi p}{p^{2}+\left(\frac{1}{2}+\tanh \sigma_{0}\right)^{2}}-\frac{\operatorname{coth} \pi p}{p^{2}+\left(1+\tanh \sigma_{0}\right)^{2}}\right. \\
& \left.-\frac{3 \operatorname{coth} \pi p}{p^{2}+\tanh ^{2} \sigma_{0}}\right]+\frac{d R}{d \sigma_{0}} .
\end{aligned}
$$

The following inditities reduce the remaining integral to elementary functions:

$$
\begin{aligned}
\tanh \pi p & =1-\frac{2}{\mathrm{e}^{2 \pi p}+1}, \quad \operatorname{coth} \pi p=1+\frac{2}{\mathrm{e}^{2 \pi p}-1}, \\
\int_{0}^{\infty} \frac{d p p}{\left(e^{2 \pi p}+1\right)\left(p^{2}+c^{2}\right)} & =-\frac{\ln c}{2}+\frac{1}{2} \psi\left(c+\frac{1}{2}\right), \\
\int_{0}^{\infty} \frac{d p p}{\left(e^{2 \pi p}-1\right)\left(p^{2}+c^{2}\right)} & =\frac{\ln c}{2}-\frac{1}{4 c}-\frac{1}{2} \psi(c),
\end{aligned}
$$


and we get:

$$
\frac{d}{d \sigma_{0}} \ln Z\left(\sigma_{0}\right)=\frac{1}{2 \cosh ^{2} \sigma_{0}}\left(\frac{1}{\tanh \sigma_{0}+1}-\frac{3}{\tanh \sigma_{0}}\right)+\frac{d R}{d \sigma_{0}}
$$

Intergation over $\sigma_{0}$ gives:

$$
\Gamma_{1-\text { loop }} \equiv \ln \frac{Z(\infty)}{Z\left(\sigma_{0}\right)}=\frac{3}{2} \ln \tanh \sigma_{0}-\frac{1}{2} \ln \frac{\tanh \sigma_{0}+1}{2}+R(\infty)-R\left(\sigma_{0}\right) .
$$

The first two terms arise from the determinants normalized by the free Klein-Gordon/Dirac operators and agree with the calculation based on the Gelfand-Yaglom method. The last two terms is the IR anomaly. Re-expressing the coordinate cutoff $R$ through the invariant cutoff according to (2.24), we find that the last three terms cancel and we are left with

$$
\Gamma_{1-\mathrm{loop}}=\frac{3}{2} \ln \tanh \sigma_{0}=\frac{3}{2} \ln \cos \theta_{0},
$$

in perfect agreement with the localization prediction (2.4), (2.8).

\section{Conclusions}

The IR anomaly, related to the singular nature of the conformal gauge, brings quantum string corrections computed in $[1,2]$ in agreement with localization predictions. We also checked that the conformal anomaly cancels in each individual expectation value, even before taking the ratio. This is an important consistency check of the underlying assumptions behind this calculation (for example, that ghosts and longitudinal modes mutually cancel in the ratio, or that the measure factors are constant and do not depend on the parameters of the problem).

One can use other parameters to build ratios of Wilson loops which are easier to compute in string theory, for instance an overall coupling to scalars as in [30]. But a really intersting problem is to carry out a complete calculation of quantum corrections for a single Wilson loop. A Wilson loop is a well-defined operator in field theory, and a holographic prescription to compute its expectation value in string theory should be unambiguously defined.

\section{Acknowledgments}

We would like to thank J. Aguilera-Damia, A. Dekel, D. Fioravanti, Yu. Makeenko, A. Tseytlin and E. Vescovi for interesting discussions. The work of D.M.-R. and K.Z. was supported by the ERC advanced grant No 341222. The work of K.Z. was additionally supported by the Swedish Research Council (VR) grant 2013-4329, by the grant "Exact Results in Gauge and String Theories" from the Knut and Alice Wallenberg foundation, and by RFBR grant 15-01-99504. 


\section{A Conformal anomalies}

Consider a second-order differential operator

$$
\mathcal{K}(\alpha)=\mathrm{e}^{2 \alpha \phi}\left(-D_{\mu} D^{\mu}+E\right),
$$

where $D_{\mu}=\partial_{\mu}+i A_{\mu}$, and $A_{\mu}$ and $E$ are $n \times n$ matrices. The bosonic fluctuation operators in (2.13), (2.9)-(2.11) can be all brought to this form. The fermionic operator (2.14), (2.12) has the standard Dirac form: ${ }^{5}$

$$
\mathcal{D}(\alpha)=\mathrm{e}^{\frac{3 \alpha \phi}{2}}\left(i \gamma^{\mu} D_{\mu}+\gamma^{3} a+v\right) \mathrm{e}^{-\frac{\alpha \phi}{2}},
$$

if we choose the basis of $2 \mathrm{~d}$ gamma-matrices to be $\gamma^{\tau}=-\tau_{2}, \quad \gamma^{\sigma}=\tau_{1}$, and $\gamma^{3} \equiv-i \varepsilon_{\mu \nu} \gamma^{\mu} \gamma^{\nu} / 2=\tau_{3}$. The paramater $\alpha$ is introduced for convenience, to interpolate between tilded $(\alpha=0)$ and untilded $(\alpha=1)$ operators. The dependence of the determinants of $\mathcal{K}(\alpha)$ and $\mathcal{D}(\alpha)$ on $\alpha$ is a textbook example of the anomaly [31,32]. Here we give a concise derivation, that closely follows [32].

The zeta-function regularized determinant of $\mathcal{K}(\alpha)$ is defined through the Mellin transform of its heat kernel:

$$
\ln \operatorname{det} \mathcal{K}=-\lim _{s \rightarrow 0} \frac{d}{d s} \frac{1}{\Gamma(s)} \int_{0}^{\infty} d t t^{s-1} \operatorname{Tr} \mathrm{e}^{-t \mathcal{K}} .
$$

Taking into account that

$$
\frac{\partial}{\partial \alpha} \operatorname{Tr} \mathrm{e}^{-t \mathcal{K}}=2 t \frac{\partial}{\partial t} \operatorname{Tr} \phi \mathrm{e}^{-t \mathcal{K}}
$$

we find that

$$
\frac{d}{d \alpha} \ln \operatorname{det} \mathcal{K}=2 \lim _{s \rightarrow 0} \frac{d}{d s} \frac{s}{\Gamma(s)} \int_{0}^{\infty} d t t^{s-1} \operatorname{Tr} \phi \mathrm{e}^{-t \mathcal{K}} .
$$

Since the gamma-function has a pole at zero, the right-hand-side seems to vanish, which would indicate that the determinant of $\mathcal{K}(\alpha)$ does not depend on the scale factor at all. But the Mellin transform generates a pole at $s=0$, because the integrand is badly behaved at $t=0$. Indeed, for any function $f(t)$ that admits a finite Laurent expansion at zero, the residue of the Mellin transform coincides with the residue of the function itself:

$$
\int_{0}^{\infty} d t t^{s} f(t) \stackrel{s \rightarrow 0}{=} \frac{1}{s} \underset{t=0}{\operatorname{res}} f(t)+\text { regular. }
$$

The small- $t$ behavior of the heat kernel is controlled by the DeWitt-Seeley expansion:

$$
\operatorname{Tr} \phi \mathrm{e}^{-t \mathcal{K}}=\sum_{k=0}^{\infty} t^{\frac{k}{2}-1} a_{k}(\phi \mid \mathcal{K}),
$$

where $a_{k}$ are local functionals of $\phi, E$ and $A_{\mu}$ that can be computed algebraically. We thus find that

$$
\frac{d}{d \alpha} \ln \operatorname{det} \mathcal{K}=2 a_{2}(\phi \mid \mathcal{K}) .
$$

\footnotetext{
${ }^{5}$ Here we assume that the connection is Abelian, and that $A_{\mu}$ is a one-component $\mathrm{U}(1)$ gauge field.
} 
The second DeWitt-Seeley coefficient of the operator (A.1) is

$$
a_{2}(\phi \mid \mathcal{K})=-\frac{1}{4 \pi} \int d^{2} \sigma\left(\frac{\alpha n}{3} \partial_{\mu} \phi \partial^{\mu} \phi+\phi \operatorname{tr} E-\frac{n}{2} \partial_{\mu} \partial^{\mu} \phi\right) .
$$

Integrating (A.7) we express the anomaly as a local functional of the fields:

$$
\ln \frac{\operatorname{det} \mathcal{K}(1)}{\operatorname{det} \mathcal{K}(0)}=-\frac{1}{2 \pi} \int d^{2} \sigma\left(\frac{n}{6} \partial_{\mu} \phi \partial^{\mu} \phi+\phi \operatorname{tr} E-\frac{n}{2} \partial_{\mu} \partial^{\mu} \phi\right) .
$$

Written that way, the anomaly does not have any boundary terms, ${ }^{6}$ but it is more natural to represent it in a different form:

$$
\ln \frac{\operatorname{det} \mathcal{K}(1)}{\operatorname{det} \mathcal{K}(0)}=\frac{1}{2 \pi} \int d^{2} \sigma\left(\frac{n}{6} \phi \partial_{\mu} \partial^{\mu} \phi-\phi \operatorname{tr} E\right)+\frac{n}{12 \pi} \oint d s\left(\phi \partial_{n} \phi-3 \partial_{n} \phi\right),
$$

where $\partial_{n}$ is the inward normal derivative at the boundary. The bulk and boundary terms in the anomaly actually arise in the computation of the Seeley coefficient exactly as written in the last expression. The more compact two-dimensional form is obtained upon integration by parts.

To compute the anomaly for fermions we first square the Dirac operator and then by the same chain of argument that led to (A.7) arrive at

$$
\frac{d}{d \alpha} \ln \operatorname{det} \mathcal{D}^{2}=2 a_{2}\left(\phi \mid \mathcal{D}^{2}\right)
$$

The square of the Dirac operator (A.2) is

$$
\begin{aligned}
\mathcal{D}^{2}(\alpha)= & \mathrm{e}^{2 \alpha \phi}\left[-\nabla_{\mu} \nabla^{\mu}+\frac{\alpha}{2} \partial_{\mu} \partial^{\mu} \phi+a^{2}-v^{2}\right. \\
& \left.+\varepsilon^{\mu \nu}\left(\partial_{\mu} a+\alpha a \partial_{\mu} \phi\right) \gamma_{\nu}+\left(\frac{1}{2} \varepsilon^{\mu \nu} F_{\mu \nu}+2 a v\right) \gamma^{3}\right]
\end{aligned}
$$

where

$$
\nabla_{\mu}=D_{\mu}-i v \gamma_{\mu}-\frac{i \alpha}{2} \varepsilon^{\mu \nu} \partial_{\nu} \phi \gamma^{3}
$$

This operator has the form (A.1) and its second DeWitt-Seeley coefficient can be read off (A.9):

$$
a_{2}\left(\phi \mid \mathcal{D}^{2}\right)=\frac{1}{4 \pi} \int d^{2} \sigma\left[\frac{\alpha}{3} \partial_{\mu} \phi \partial^{\mu} \phi+2 \phi\left(v^{2}-a^{2}\right)-\alpha \partial_{\mu}\left(\phi \partial^{\mu} \phi\right)+\partial_{\mu} \partial^{\mu} \phi\right] .
$$

We thus find for the fermion anomaly:

$$
\begin{aligned}
\frac{1}{2} \ln \frac{\operatorname{det} \mathcal{D}^{2}(0)}{\operatorname{det} \mathcal{D}^{2}(1)}= & \frac{1}{2 \pi} \int d^{2} \sigma\left[\frac{1}{12} \phi \partial_{\mu} \partial^{\mu} \phi+\phi\left(a^{2}-v^{2}\right)\right] \\
& -\frac{1}{12 \pi} \oint d s\left(\phi \partial_{n} \phi-3 \partial_{n} \phi\right) .
\end{aligned}
$$

Notice that the boundary anomaly has the same magnitude but different sign compared to bosons.

\footnotetext{
${ }^{6}$ Here we assume that the metric is flat and the boudary is straight. These simplifying assumptions are sufficient for our analysis. Otherwise curvature also contributes to the anomaly.
} 
Open Access. This article is distributed under the terms of the Creative Commons Attribution License (CC-BY 4.0), which permits any use, distribution and reproduction in any medium, provided the original author(s) and source are credited.

\section{References}

[1] V. Forini, V. Giangreco M. Puletti, L. Griguolo, D. Seminara and E. Vescovi, Precision calculation of 1/4-BPS Wilson loops in $A d S_{5} \times S^{5}$, JHEP 02 (2016) 105 [arXiv: 1512.00841] [INSPIRE].

[2] A. Faraggi, L.A. Pando Zayas, G.A. Silva and D. Trancanelli, Toward precision holography with supersymmetric Wilson loops, JHEP 04 (2016) 053 [arXiv: 1601.04708] [INSPIRE].

[3] V. Forini, A.A. Tseytlin and E. Vescovi, Perturbative computation of string one-loop corrections to Wilson loop minimal surfaces in $A d S_{5} \times S^{5}$, JHEP 03 (2017) 003 [arXiv: 1702.02164] [INSPIRE].

[4] J.M. Maldacena, Wilson loops in large-N field theories, Phys. Rev. Lett. 80 (1998) 4859 [hep-th/9803002] [INSPIRE].

[5] S.-J. Rey and J.-T. Yee, Macroscopic strings as heavy quarks in large- $N$ gauge theory and anti-de Sitter supergravity, Eur. Phys. J. C 22 (2001) 379 [hep-th/9803001] [InSPIRE].

[6] N. Drukker, D.J. Gross and H. Ooguri, Wilson loops and minimal surfaces, Phys. Rev. D 60 (1999) 125006 [hep-th/9904191] [INSPIRE].

[7] J.K. Erickson, G.W. Semenoff and K. Zarembo, Wilson loops in $N=4$ supersymmetric Yang-Mills theory, Nucl. Phys. B 582 (2000) 155 [hep-th/0003055] [inSPIRE].

[8] N. Drukker and D.J. Gross, An Exact prediction of $N=4$ SUSYM theory for string theory, J. Math. Phys. 42 (2001) 2896 [hep-th/0010274] [INSPIRE].

[9] V. Pestun, Localization of gauge theory on a four-sphere and supersymmetric Wilson loops, Commun. Math. Phys. 313 (2012) 71 [arXiv:0712.2824] [inSPIRE].

[10] K. Zarembo, Localization and AdS/CFT Correspondence, J. Phys. A 50 (2017) 443011 [arXiv: 1608.02963] [INSPIRE].

[11] N. Drukker, D.J. Gross and A.A. Tseytlin, Green-Schwarz string in $A d S_{5} \times S^{5}$ : Semiclassical partition function, JHEP 04 (2000) 021 [hep-th/0001204] [INSPIRE].

[12] M. Kruczenski and A. Tirziu, Matching the circular Wilson loop with dual open string solution at 1-loop in strong coupling, JHEP 05 (2008) 064 [arXiv:0803.0315] [INSPIRE].

[13] C. Kristjansen and Y. Makeenko, More about One-Loop Effective Action of Open Superstring in $A d S_{5} \times S^{5}$, JHEP 09 (2012) 053 [arXiv: 1206.5660] [INSPIRE].

[14] R. Bergamin and A.A. Tseytlin, Heat kernels on cone of $A d S_{2}$ and $k$-wound circular Wilson loop in $A d S_{5} \times S^{5}$ superstring, J. Phys. A 49 (2016) 14LT01 [arXiv:1510.06894] [INSPIRE].

[15] N. Drukker, 1/4 BPS circular loops, unstable world-sheet instantons and the matrix model, JHEP 09 (2006) 004 [hep-th/0605151] [INSPIRE].

[16] N. Drukker, S. Giombi, R. Ricci and D. Trancanelli, More supersymmetric Wilson loops, Phys. Rev. D 76 (2007) 107703 [arXiv:0704.2237] [inSPIRE].

[17] V. Pestun, Localization of the four-dimensional $N=4 S Y M$ to a two-sphere and $1 / 8$ BPS Wilson loops, JHEP 12 (2012) 067 [arXiv:0906.0638] [INSPIRE]. 
[18] K. Zarembo, Supersymmetric Wilson loops, Nucl. Phys. B 643 (2002) 157 [hep-th/0205160] [INSPIRE].

[19] S.-x. Chu, D. Hou and H.-c. Ren, The Subleading Term of the Strong Coupling Expansion of the Heavy-Quark Potential in a $N=4$ Super Yang-Mills Vacuum, JHEP 08 (2009) 004 [arXiv:0905.1874] [INSPIRE].

[20] V. Forini, Quark-antiquark potential in AdS at one loop, JHEP 11 (2010) 079 [arXiv: 1009.3939] [INSPIRE].

[21] X. Chen-Lin, D. Medina-Rincon and K. Zarembo, Quantum String Test of Nonconformal Holography, JHEP 04 (2017) 095 [arXiv:1702.07954] [INSPIRE].

[22] N. Drukker and B. Fiol, On the integrability of Wilson loops in $A d S_{5} \times S^{5}$ : Some periodic ansatze, JHEP 01 (2006) 056 [hep-th/0506058] [INSPIRE].

[23] J. Greensite and P. Olesen, World sheet fluctuations and the heavy quark potential in the AdS/CFT approach, JHEP 04 (1999) 001 [hep-th/9901057] [INSPIRE].

[24] S. Förste, D. Ghoshal and S. Theisen, Stringy corrections to the Wilson loop in $N=4$ super Yang-Mills theory, JHEP 08 (1999) 013 [hep-th/9903042] [INSPIRE].

[25] Y. Kinar, E. Schreiber, J. Sonnenschein and N. Weiss, Quantum fluctuations of Wilson loops from string models, Nucl. Phys. B 583 (2000) 76 [hep-th/9911123] [INSPIRE].

[26] V. Forini, V.G.M. Puletti, L. Griguolo, D. Seminara and E. Vescovi, Remarks on the geometrical properties of semiclassically quantized strings, J. Phys. A 48 (2015) 475401 [arXiv: 1507.01883] [INSPIRE].

[27] W.I. Weisberger, Conformal Invariants for Determinants of Laplacians on Riemann Surfaces, Commun. Math. Phys. 112 (1987) 633 [InSPIRE].

[28] A.A. Abrikosov, L.P. Gorkov and I.E. Dzyaloshinski, Methods of quantum field theory in statistical physics, Prentice-Hall (1963).

[29] N. Rosen and P.M. Morse, On the vibrations of polyatomic molecules, Phys. Rev. 42 (1932) 210.

[30] M. Beccaria, S. Giombi and A. Tseytlin, Non-supersymmetric Wilson loop in N=4SYM and defect $1 d$ CFT, arXiv:1712.06874 [INSPIRE].

[31] A.S. Schwarz, Quantum field theory and topology, Springer (1993).

[32] D. Fursaev and D. Vassilevich, Operators, geometry and quanta, Springer (2011). 